\title{
XAVIER ZUBIRI FRENTE A LA LÓGICA \\ MODERNA: LOGICISMO, FORMALISMO \\ E INTUICIONISMO LÓGICOS
}

\section{ZUBIRI AND THE MODERN LOGIC}

\author{
Jesús Ramírez Voss \\ Fundación Xavier Zubiri
}

\begin{abstract}
RESUMEN: Este artículo recoge la invitación del profesor Vega Reñón ${ }^{1}$ a investigar la recepción de la moderna lógica simbólica o matemática en España entre 1890 y 1930. Recepción que considera lograda entre los matemáticos pero lamentablemente fallida entre los filósofos españoles contemporáneos. Examinamos aquí en detalle la recepción que de la lógica formal contemporánea llevó a cabo Xavier Zubiri comenzando desde sus primeros escritos de doctorado hasta su labor docente en la cátedra de la universidad Central de Madrid en los años treinta del pasado siglo XX. Interesa aquí pues la recepción llevada a cabo por Zubiri del intuicionismo de Brouwer, del formalismo de Hilbert y del logicismo de B.Russell,
\end{abstract}

DESCRIPTORES: Lógica formal, Filosofía española del siglo XX, Zubiri, Intuicionismo, Formalismo de Hilbert, Logicismo de Russell.

ABSTRACT: This paper is a second step towards the study of the reception of modern — symbolic, mathematical — logic in Spain. An invitation to go on by Professor Vega Reñón. His works show us an unsuccessful introduction of modern logic in philosophical circles of Spanish contemporary

\footnotetext{
${ }^{1}$ Vega Reñón, L.: «La lógica en España (1890-1930): desencuentros». Teorema. Revista internacional de Filosofia. VOL XX/1-2, 2001; pp. 21-38.
} 
philosophers. We examine the reception of formal logic by Xavier Zubiri from his early works until his teaching at Central University in Madrid during the thirties years of last twenty century. We focused this problem on Brower's intuitionism, Hilbert's formalism and Russell's logicism.

KEYWORDS: Formal logic, $20^{\text {th }}$ century Spanish philosophy, Zubiri, intuitionism, formalism, logicism, schools of though in mathematical logic.

\section{Introducción}

No parece que a primera vista la filosofía de Xavier Zubiri, centrada en la descripción de lo real actualizado en inteligencia sentiente, tenga en consideración suficiente las distintas concepciones de la lógica formal contemporánea. Pero no es así. Desde su tesis de doctorado hasta sus primeros cursos como catedrático en Madrid, Zubiri dio buena cuenta de las diferentes corrientes en que aparecía la nueva lógica. $Y$ este preciso aspecto es lo que otorga interés al presente artículo. Se trata de una puesta al día, por así decir, de las fuentes bibliográficas que posibilitaron o que desviaron la recepción y contenidos de la lógica formal contemporánea en el pensamiento español de la primera mitad del siglo pasado. Es cierto que este asunto, la lógica formal contemporánea, apenas había sido tratado ni en la escasa obra publicada por Zubiri en vida, ni, todavía hoy, entre los estudios más conocidos acerca de Zubiri. Sin embargo, no por ello se trata de un problema menor o secundario. Tengo el vivo convencimiento de que no es así. No fue la lógica formal, de ninguna manera, un fondo profundo que quedara inexplorado por el autor de Sobre la esencia. Zubiri mismo reconoció su afán por la lógica; varios trabajos suyos dan hoy buena cuenta de ello: su tesina de licenciatura ${ }^{2}$ dedicada al problema de la objetividad en la fenomenología de Edmund Husserl y el proyecto de una lógica pura. Era el año veintiuno (1921). Décadas más tarde, en plena madurez intelectual y vital, Zubiri en el Prólogo a la edición inglesa de Naturaleza, Historia, Dios ${ }^{3}$ declara abiertamente que su propósito como pensador fue

2 Zubiri, X.: Primeros Escritos (1921-1926). Alianza Editorial Fundación Xavier Zubiri. Madrid, 1999; pp. 1-60. En adelante se cita como PE y página correspondiente.

3 Zubiri, X.: Naturaleza, Historia, Dios. Alianza Editorial Fundación Xavier Zubiri. Madrid, 1987. En adelante NHD. 
nada más y nada menos que la afanosa búsqueda de una lógica de la realidad. Era el año ochenta (1980). Por lo tanto, la lógica formal marcó necesariamente una decidida impronta para el autor de la trilogía sobre la inteligencia sentiente.

\section{La lógica formal}

Primero de todo entonces ¿Qué entender de modo genérico por lógica formal? Naturalmente no se trata de comprimir ahora el desarrollo histórico completo de esta disciplina, comenzando por los tratados aristotélicos que componen el Organon así como las aportaciones de megáricos y estoicos. Cabe recordar de modo muy sintético que, sencillamente, a la teoría formal de la deducción, tal y como fue cultivada desde Aristóteles a Leibniz, se le da el nombre genérico de lógica formal. Es aquella ciencia de la que afirmaba Kant en su Prólogo ${ }^{4}$ a la segunda edición de la Crítica de la razón pura que se hallaba definitivamente completa y concluida pues había tomado el camino seguro de la ciencia desde los griegos. Según Kant, la lógica formal tan sólo había necesitado alguna sutileza que afectaba más a su exposición externa que a la certeza de su formalidad. Kant consideraba que la lógica formal se hallaba acertadamente llegada a su fin.

No fue solamente esta la concepción de la lógica a la que tuvo que confrontar Zubiri la elaboración de su propio sistema de pensamiento. Al menos no fue esta concepción lógica formal la que trató como auténtico problema filosófico. La ciencia que Kant consideraba completa y acabada desde los Analíticos Primeros de Aristóteles, decididamente no puede considerarse sin más como la Lógica Formal pero tampoco resulta incompatible con la concepción contemporánea de la lógica, aun sabiendo lo muy discutido de este asunto entre algunos historiadores. Un clásico en la discusión, duraqnte los años treinta, fue el artículo de Rudolf Carnap 5 en el primer número de la revista Erkenntnis, diferenciando entre una antigua y una moderna lógica. La frontera entre una y otra lógica era el sometimiento del razonamiento formal a la matematización.

${ }^{4}$ Kant, I.: Crítica de la razón pura .Ediciones Alfaguara. Prólogo, Traducción, Notas e Índices de Pedro Rivas. Madrid, 1978. BVIII.

${ }^{5}$ Véase AyER, A. J.: El positivismo lógico. F.C.E. México, 1965; pp. 139-152. Asimismo, MuÑoz Delgado, V.: Lógica matemática y lógica filosófica. Madrid, 1962. 


\section{Lógica formal contemporánea}

Consecuentemente, la así llamada lógica formal contemporánea dio comienzo de manera simultánea en diferentes momentos y se fue delimitando en diferentes corrientes ${ }^{6}$. La obra de G. Frege, Conceptografía fue, no cabe duda, un prodigioso paso inicial a pesar de que el exceso de complicación en su escritura simbólica, resultara un factor decisivo que determinó su escasa difusión. Seguramente Zubiri no conoció esta obra. Y junto con Frege, destacaron Bernardo Bolzano, G. Boole, Auguste De Morgan etc formidables pensadores dedicados al desarrollo de la lógica a finales del siglo diecinueve. Además, resultó de capital influencia para el avance de la moderna lógica formal matematizada la aparición de los Fundamentos para una teoría general de conjuntos llevada a cabo por el matemático germano G. Cantor. Esto último, marcó de nuevo un decidido interés por la matemática muy ajustada a los intereses de la filosofía de Xavier Zubiri.

La matematización de la lógica tuvo como resultado un mayor y más perfecto control formal en la formulación de los razonamientos, un conocimiento más preciso de las leyes del pensamiento y, lo más importante y fecundo de todo, el descubrimiento de nuevos y variados sistemas de inferencia que, de otro modo, hubiera sido casi imposible manejar. Todo esto quedo plasmado en la base de una de las más famosas obras de lógica del siglo XX: Principia Matemática (19101913) cuyos autores fueron Alfred N. Whitehead y Bertrand Russell. A esta concepción de la lógica formal matematizada se le han dado los nombres de álgebra lógica, lógica simbólica y logistica. Este último término, por cierto, a propuesta de Couturat en el Congreso Internacional de Filosofía, celebrado en Ginebra en 1904. Y logística fue el término que también, para referirse a la lógica formal contemporánea utilizaron primero Ortega y Gasset y luego García Bacca o Manuel Granel y así, con esa denominación, se refiere a ella Zubiri en su tesis de doctorado Ensayo de un teoría fenomenológica del Juicio. Era el año veintitrés (1923). Zubiri deja escrito que la filosofía contemporánea tiene su raíz en una interpretación objetivista de la matemática y que Husserl, partiendo del psicologismo de Brentano, ha intentado escribir una Filosofía de la Aritmética llegando luego en

${ }^{6}$ Vega Reñón, L.: Una guía de historia de la Lógica. U.N.E.D. Madrid, 1996. Cap. 6 Lógica Contemporánea; pp. 186-209. 
sus Investigaciones lógicas a un objetivismo puro, completado por la idea de una fenomenología. Y añade:

«El movimiento traspasa las fronteras germánicas. En Inglaterra, Russell, a base de las matemáticas y de la teoría de Meinog, orienta el pensamiento inglés hacia la lógica matemática y una definición realista de la verdad. De ahí pasa la corriente a Francia y toma cuerpo en la logística de Couturat ${ }^{7} »$.

Como se puede comprobar, tenemos ahí la primera referencia explícita de Zubiri al asunto que nos concierne. El jovencísimo filósofo estaba ya al tanto de las novedosas publicaciones en torno a esta disciplina, la logística. ¿Qué juicio le mereció? ¿Qué alcance tuvo en su formación como filósofo? ¿Qué repercusiones se pueden hallar en su trayectoria posterior? ¿Qué objeciones señaló de ella? Todas estas preguntas salen a la luz por primera vez en este artículo de modo que se ponen a partir de ahora en discusión pública en la obra de Zubiri. Después de Russell, en la década de los años veinte cobró particular importancia la obra lógica de L.E.J. Brouwer y D. Hilbert, creadores, respectivamente, de la corriente intuicionista y formalista de la lógica contemporánea. Ambas estuvieron centradas en el ámbito de problemas acerca de la fundamentación de la matemática. De ambos también da cuenta el joven su Zubiri:

«Brouwer y Weil nos presentan una interpretación casi finitista e intuitiva de los conjuntos, audaz concepción que viene a poner en crisis nociones tan cardinales como la de infinito actual ${ }^{8}$ ".

"Mediante la teoría de los grupos, la Matemática moderna ha obtenido el fin que perseguía. Se han sistematizado todas las teorías del análisis, algebraico y transcendente [...] ¿Quién pone estas condiciones? A veces la experiencia; otras veces, lo más frecuente es la razón. Se comienza por definir ciertas relaciones fundamentales y se deducen sus consecuencias bajo la sola condición de conservar sus propiedades formales. El método intuitivo ha sido sustituido por el método axiomático (Hilbert) ${ }^{9}$ ».

7 PE. , 113 .

${ }^{8} \mathrm{PE}, 342$.

${ }^{9} \mathrm{PE}, 97$. 
A través de estas citas se revela claramente que, ya a la altura de los años veinte del pasado siglo, el joven Zubiri contaba con un repertorio de lecturas adecuado y parece que bastante completo acerca de la lógica formal contemporánea: conocía el logicismo de Russell, el intuicionismo de Brouwer y el formalismo de Hilbert. Se trata ahora de analizar la recepción y el alcance de cada una de ellas pareja a la formación de Zubiri como filósofo.

\section{Zubiri y el problema de la logística}

Como es sabido, Russell ${ }^{10}$ defendió la tesis fundamental de que la Matemática y la lógica son idénticas. Una tesis que él mismo reconoció que resultaba muy impopular. Impopular para los matemáticos, porque la lógica tradicionalmente se asociaba con la filosofía y, en concreto con la filosofía de Aristóteles, de manera que los matemáticos consideraban que la lógica no era disciplina de su incumbencia. Y así mismo impopular para los filósofos y lógicos aristotélicos, porque se mostraban ofendidos o molestos cuando se les pedía que utilizasen una técnica de simbolismo matemático nueva y muy difícil de manejar.

¿Fue ese el obstáculo que encontró Zubiri en la recepción de la logística?:Fue la complejidad matemática del simbolismo lógico el problema que estamos investigando aquí? Mi respuesta es que no. No fue un problema de dificultad con respecto al manejo del simbolismo matemático lo que impidiera en Zubiri una asimilación de la lógica matemática russelliana. La razón es la siguiente: Zubiri estudió matemáticas con Barinaga y con Rey Pastor en Madrid. Se conservan unos apuntes de Logística y Psicología ${ }^{11}$ del año diecisiete (1917) y numerosos estudios sobre cuestiones matemáticas, por ejemplo: La evolución de la matemática en el edad contemporánea ${ }^{12}$ de ese mismo año 1917. Y desde su tesis de doctorado ${ }^{13}$ Zubiri advertía ya de la ausencia en nuestras Faculta-

${ }^{10}$ Russell, B.: Los principios de la Matemática. Traducción de J. C. Grimberg. Espasa-Calpe. Madrid, 1983 (Introducción a la segunda edición); pp. 7 y ss.

${ }^{11}$ Archivo Xavier Zubiri. Fundación Xavier Zubiri. Madrid. Signatura 0002009 Contenido: Apuntes de Logistica y Psicología, 1917. En adelante citamos simplemente como Archivo.

12 Archivo SIGNATURA (II) Evolución de la matemática contemporánea. Madrid, 1916, p. 31 .

${ }^{13} \mathrm{PE}, 68-317$ (La tesis se titula Ensayo de una teoría fenomenológica del juicio). 
des de Filosofía de una especialidad como Filosofía de la Matemática. Es, entonces, en este amplio contexto en el que resulta pertinente centrarse en una de las fuentes que el mismo Zubiri maneja, me refiero al texto de Rey Pastor ${ }^{14}$ que aparece citado en su tesis. En efecto, en este breve tratado de matemáticas se describe el estado de la disciplina, sus problemas y métodos más actuales entonces, desde los fundamentos de la Aritmética y el Análisis hasta las dificultades halladas en el campo de la Geometría. Interesa resaltar que Rey Pastor, del mismo modo que luego el joven Zubiri, encara el alcance de la logística russelliana, situándola junto con el formalismo de Hilbert y junto con el intuicionismo de Hilbert. No hay prioridad ni relevancia de uno sobre los demás. El logicismo del gigante Russell, así lo llama Rey Pastor, no sirve más que de orientación en los modernos desarrollos de la matemática y, por lo tanto, no es al único lógico a quien se le estudia o se le tiene en cuenta. Insisto, no parece que una posible falta de preparación en el simbolismo matemático fuera para Zubiri el problema de la lógica formal contemporánea. Por lo tanto, si descartamos una deficiente formación matemática, sería conveniente investigar su formación en lógica.

El joven Zubiri cuenta, entre la bibliografía de su tesis de doctorado con un ensayo muy interesante, bastante extenso y formativo acerca de lo que, en los medios filosóficos españoles de principios del siglo pasado, se daba a entender por lógica formal. Me refiero al manual del catedrático de lógica de la Universidad de Granada ${ }^{15}$. Me parece que este libro, sepultado en el completo olvido, respondía a la irrupción de la nueva lógica matemática, que él denomina lógica algorítmica, desde una posición neoescolástica abierta y rigurosa. Considera Gómez Izquierdo que el legado lógico aristotélico se ha diversificado en diferentes corrientes o direcciones: una es la que denomina dirección idealista en la que incluye a autores tan alejados hoy de la lógica formal como Fichte o Krause. Otra dirección es la que denomina Lógica inductiva en la que incluye a Spencer y Stuart Mill: una tercera dirección se dirige hacia los textos de Wundt y Sigwart y que denomina lógica de las ciencias. Finalmente, la dirección que resulta relevante para nuestro asunto aquí, es la que el catedrático granadino denomina sin más lógica formal y que está dedicada sobre todo a la cuantifica-

\footnotetext{
${ }^{14}$ Rey PASTOR, J.: Introducción a la matemática superior. Editorial Corona. Madrid, 1916.

15 Gómez IzQuierdo, A.: Nuevas direcciones de la lógica. Librería General de Victoriano Suárez. Madrid, 1917.
} 
ción de los predicados llevada a cabo por Hamilton pero, sobre todo, la lógica algoritmica, cuyo principal representante para Gómez Izquierdo no es Russell sino Boole. Su consideración acerca de esta nueva dirección en lógica es la siguiente:

«La lógica algorítmica se ha fijado principalmente en el aspecto cuantitativo de las nociones y en el valor positivo y negativo de los juicios, pues no es fácil someter a signos tan sencillos y uniformes como los matemáticos las múltiples ideas y relaciones que por los actos mentales concebimos. Además, como ya he advertido, nuestros juicios implican de un modo primordial y directo relaciones de compresión, y estas son la base y funcionamiento de las relaciones de extensión. A lo sumo, la lógica algorítmica podrá ser un ensayo de ingenio, una curiosidad científica, pero sin finalidad práctica; y si nadie, que sepamos, se ha decidido a emplearla en sus investigaciones, mucho menos podrá esperarse que sustituya a lógica tradicional» ${ }^{16}$.

Por lo tanto, la consideración del profesor granadino es que la novísima lógica formal es un instrumento científico simbólico que apenas nadie utiliza y que resulta muy poco probable que sustituya a la lógica tradicional, de base aristotélica y, en un par de notas a pie de página añade que además esta lógica algebraica exige un caudal de conocimientos matemáticos muy complicados de exponer, así como un lenguaje artificial enmarañado de signos completamente artificiosos - justamente, lo que señalaba Russell en su introducción-. Con este criterio tan desalentador no parece que invitara a nadie a seguir cultivando esta disciplina. Pero esto no es todo. De este mismo autor, Gómez Izquierdo, recuerdo que era catedrático de lógica en la universidad de Granada, el joven Zubiri tenía (y aún se conserva) otro manual posterior, del año veintiocho (1928) titulado Análisis del pensamiento lógico ${ }^{17}$. Este manual amplía y reconoce un alcance distinto de la moderna lógica formal. En efecto, aparecen los nombres de Frege (aunque no hay referencia a ninguna de sus obras), Couturat (L'Algebre de la Loqique), Boole (An investigation of the Laws of Thought) y por supuesto, Russell

16 Oc., p. 46.

17 Gomez IzQuierdo, A.: Análisis del pensamiento lógico. VOL .I. El concepto y la palabra .La definición y la división. Facultad de Filosofía y Letras Universidad de Granada.1928. El libro que se conserva en la biblioteca personal de Zubiri contiene la siguiente dedicatoria: «joven maestro de filosofía y amigo queridísimo». 
y Whitehead (Principia Matemática) así como Peano y Schröder. Todos ellos hacen del logicismo:

«un formalismo absoluto de carácter matemático (...) una técnica nueva de exposición y demostración (...) una simbólica universal, en donde el encadenamiento de las fórmulas carece de verdad y de significación intrínseca: su característica, según Russell "ni sabe de lo que se habla, ni si lo que se dice es verdadero". Este puro formalismo y mecanismo intelectuales trae a la memoria el sueño de Raimundo Lulio en su Ars Magna ${ }^{18}$.

Observando así los textos, revisadas cada una de las principales fuentes, tenemos que la logística es:

a) sólo una más de las diferentes direcciones en que marcha la lógica

b) un movimiento característico principalmente de Inglaterra, Boole, Russell

c) una disciplina que exige un conocimiento preciso y molesto del simbolismo matemático

d) un artilugio intelectual rebuscado que no utiliza nadie

e) una disciplina que carece de verdad y de significación intrínseca y

f) un formalismo cuyo origen se remonta a Raimundo Lulio.

La huella de estos novísimos estudios de lógica formal quedó, a mí me parece que de una forma bien clara, en el joven Zubiri. El joven filósofo compartió de modo general la crítica y el desinterés que el profesor granadino mostraba en sus manuales con respecto a la logística, la moderna dirección de la lógica contemporánea llevada a cabo por Russell. Aquí sí que hallamos el primer aspecto del problema que presenta la logística:

«No seré yo quien niegue valor a esta audaz concepción [de la lógica] perseguida con anhelo desde Leibniz y quizá desde Raimundo Lulio: tiene la indiscutible ventaja de sistematizar rigurosamente esta difícil parte de la

${ }^{18}$ Oc., p. 67. 
Lógica. Tan sólo habré de excluir mi adhesión en el caso, nada raro, de querer hacer de la logística toda la lógica; porque por grande que sea la utilidad del simbolismo, no habrá de olvidarse nunca que su valor es de pura utilidad y no de verdad ${ }^{19}$.

Tenemos, por tanto, dos importantes objeciones: la primera es querer hacer de la logística la totalidad de la Lógica y la segunda es que el valor del simbolismo no es de verdad sino de mera utilidad. Con respecto a la primera objeción, Zubiri compartiría el punto de vista del matemático Rey Pastor: la logística no es sin más la lógica, caben al menos tres formulaciones más, la aristotélica, la intuicionista y la formalista. Reconoce el joven Zubiri que la logística no es un simbolismo inútil, sino que proporciona rigor a esta difícil parcela del saber. En cualquier caso, son objeciones de menor calado filosófico por lo que respecta a la cuestión final: la logistica no proporciona un acceso a la verdad. Aquí reside el auténtico problema de esta nueva disciplina filosófica. La verdad, afirma Zubiri, no tiene su sitio en el simbolismo matemático formal de las proposiciones lógicas.

En efecto, lo característico de un lenguaje lógicamente perfecto como el que querían conseguir Russell y Whitehead, es tratar de cumplir con un principio de extensionalidad: todas las proposiciones complejas del lenguaje lógico habrían de descomponerse en otras simples o atómicas, de tal manera que la verdad o falsedad de las primeras fuera una función de verdad de las segundas. $Y$ ¡cuál es este valor de verdad? La respuesta de Russell es que una proposición atómica se corresponde con un hecho del mundo. Un hecho es, simplemente, aquello que hace verdadera o falsa una proposición atómica. Y una proposición compleja no es en sí misma ni verdadera ni falsa sino en función de las obras que lo componen. Por lo tanto, la primera condición para que un lenguaje sea lógicamente perfecto es una condición lingüístico-formal: que las palabras de cada proposición simple se correspondan una por una a los componentes del hecho correspondiente, exceptuando términos tales como $\mathrm{y} / \mathrm{o} / \mathrm{no} / \mathrm{si}$...entonces que tienen una función diferente, la de conectar entre sí las distintas proposiciones simples. Un lenguaje semejante, afrimaba Russell, tiene la ventaja de que muestra a simple vista la estructura lógica de los hechos que afirma o niega. Principia Matemática ejemplificaba ese modelo de lenguaje, un conjunto de símbolos, un cálculo lógico que,

${ }^{19}$ PE, 327. 
en virtud de cierto vocabulario y sintaxis nos hubiera evitado todos los errores en los que ha incurrido la filosofía tradicional.

Me parece que el joven Zubiri vio en este intento una empresa lógico-filosófico difícilmente compatible con una teoría de la verdad que se correspondiera con la realidad de las cosas, por mucho que Russell se refiera a los hechos del mundo. La crítica de Zubiri se encuentra en lo siguiente:

«En su proceso simbólico, la logística se inspira en la teoría gramatical de los elementos del juicio; esto es, considera que el sujeto es el sujeto de la oración, que el predicado es el complemento y que la cópula expresa relación. Pero ya sabemos que esto no es así. En la relación hay dos términos y la relación misma; la síntesis de estos tres elementos es la unidad de la relación. El sujeto no es uno de los términos de la relación sino la síntesis de todos ellos; el predicado es el pensamiento de la relación, que es referido intencionalmente al sujeto en virtud de la cópula. En estos juicios hay, pues, una doble relación: la de sujeto a predicado (común a todo juicio) y la existente dentro del sujeto entre los dos relatos. La logística no simboliza sino uno de sus posibles objetos. No puede, pues, aspirar a una Lógica universal ${ }^{20}{ }$.

No se trata, pues, de realizar una nueva gramática filosófica por muy matematizada que se nos muestre, porque no nos asegura ni la verdad de las cosas ni la adecuada relación entre el lenguaje, el pensamiento y las cosas. Aunque Zubiri emplea el término juicio frente al de proposición, el fondo de su crítica es, para su propio proyecto filosófico, definitivo, porque ya se trate de juicios de la forma SesP ya de trate de proposiciones cuantificadas y simbolizadas matemáticamente, lo cierto es que no despegamos nuestro análisis de las cosas de la estructura gramatical del lenguaje natural que empleamos bajo su simbolismo formal. Este es el problema radical de todo lenguaje lógico, sobre todo cuando el propósito es nada más y nada menos que el de elaborar un lenguaje perfecto. El joven Zubiri es rotundo: la logística no puede convertirse en modo alguno en un renovado lenguaje universal. Con independencia de las paradojas a que posteriormente diera lugar, sin duda ninguna, auténticas aporías en el caso del proyecto de Principia Mathematica, para Zubiri — lo mismo que para Gómez Izquierdola logística se ha estancado en el proceso de análisis del lenguaje, teoría gramati-

\footnotetext{
${ }^{20} \mathrm{PE}, 326$.
} 
cal de elementos del juicio - $-\mathrm{y}$ su simbolismo matemático carece de auténtico valor de verdad-.

Hasta donde sabemos, Zubiri jamás volvió a referirse a Russell y a la logística a lo largo de toda su longeva trayectoria intelectual. No le interesó. Y con respecto a las verdades de la lógica rechazó siempre la distinción entre verdades de razón /verdades de hecho propuesta por Leibniz ${ }^{21}$ pues todas las verdades lo son siempre de la realidad. Un concepto como el de verdad lógica o tautología carece de sentido principal, frente a la verdad real en el ámbito metafísico de la inteligencia sentiente. Para concluir este apartado, recuerdo simplemente que:

«lo lógico de la verdad racional consiste formalmente en cumplimiento histórico; y lo histórico de la verdad racional consiste formalmente en encuentro lógico. Es la identidad radical de lo lógico y lo histórico en toda intelección racional ${ }^{22}$.

\section{Intuicionismo lógico: Brower y Zubiri}

He encontrado que el interés de Zubiri por la lógica contemporánea no desapareció a pesar de las objeciones en contra del logicismo. Hay en el Archivo una serie de escritos inéditos sobre este asunto de la nueva lógica formal. En primer lugar, existe una Memoria de Cátedra ${ }^{23}$, del año veintiséis (1926) que tiene el siguiente título: Los problemas de la lógica contemporánea. Se trata de un trabajo que el joven Zubiri preparó con vista a las oposiciones a la cátedra de Lógica y Teoría del Conocimiento de la universidad de Salamanca. Según parece, a esta misma plaza quiso opositar también Joaquín Xirau, filósofo catalán, compañero de Zubiri durante el año de doctorado en Madrid y discípulo así mismo de Jaume Serra Hunter. Este inédito de Zubiri presenta un esquema expositivo muy completo sobre las concepciones y temas de lógica en general. Las fuentes biblio-

${ }^{21}$ ZUBIRI, X.: Inteligencia Sentiente. Inteligencia y Razón. Alianza Editorial Fundación Xavier Zubiri. Madrid, 1983; pp. 279 y ss. En adelante, IRA y número de página de esta edición.

${ }^{22}$ IRA, 306.

23 ARCHIVO Signatura 0011002 Contenido ZUBIRI, X. Los problemas de la lógica contemporánea. Texto manuscrito, calificado de nuestra memoria, que según Índice antepuesto a la misma debía tener dos partes pero parece incompleto. 
gráficas básicas con las que trabaja son prácticamente las mismas que las utilizadas en su tesis de doctorado. Lo que me interesa aquí destacar es un Apéndice mecanografiado cuyo título es el siguiente: Modernas orientaciones de los estudios lógicos. Nuestro filósofo ofrece un panorama acotado de las diferentes fundamentales de la filosofía contemporánea dentro de la lógica. Este panorama le resulta una tarea previa necesaria para orientar su pensamiento propio. Lo primero que se encuentra es todo un capítulo dedicado a la separación entre ambas disciplinas, lógica y conocimiento. Sostiene Zubiri que cualquier manual contemporáneo de lógica acepta la siguiente división básica: una teoría del conocer [Elementarlehre] y una teoría de los métodos del conocimiento [Methodenlehre]. Entre los autores que se citan destacan Sigwart y Benno Edermann. A continuación el joven opositando reitera, como ya hiciera en otros trabajos anteriores, su vínculo con la filosofía fenomenológica de Edmund Husserl, en concreto Investigaciones lógicas. Señala además la Filosofía de la Aritmética en tanto que interpretación psicológica de los conceptos fundamentales de la matemática. A primera vista, ninguna cosa que no sepamos ya salvo que he encontrado en este inédito una parte bien interesante para esta investigación. En efecto, dentro de Modernas orientaciones de los estudios lógicos se encuentra un breve capítulo dedicado a la lógica matemática. Lo interesante es que ha desaparecido cualquier alusión al logicismo, ya se trate de Boole, de Couturat o de Russell. Estos autores ya no se citan ni se tiene en cuenta la concepción de la lógica que representan; el interés de Zubiri recae ahora en el intuicionismo lógico representado por el matemático holandés Brouwer y su discípulo Weil.

Siguiendo a Poincaré y también a Boutrux, el intuicionismo sostiene que la matemática es independiente de su expresión lógico simbólica con la que podamos representarla. Casi en los mismos años de la publicación de Principia Matemática, el intuicionismo de Brouwer rechazaba por completo la tesis defendida por el logicismo; la matemática no es estructura lógico-simbólica, sino que la matemática es creación de manera que con la lógica se demuestra pero con la intuición se inventa. Brouwer afirmaba rotundo que la matemática es libre construcción del pensamiento, sin reducción posible al mundo objetivo de las ideas platónicas o al lingüístico-formal del simbolismo lógico.

¿Qué le interesó al joven Zubiri de esta nueva orientación de los estudios lógicos? Mi respuesta es que, al revés que el logicismo, el intuicionismo resultó una dirección de la lógica formal contemporánea que le pareció compatible con 
la filosofía fenomenológica de Husserl. El enlace, claro está, era el concepto de intuición. Escribe Zubiri:

«La intuición de la que hablan estos modernos críticos [por Brouwer y Weil] no es la percepción sensible, sino la intuición del ideal fenomenológico ${ }^{24}$ ».

La intuición es el carácter intelectivo del acto intencional de la conciencia [Wessenschau]. Es la aprehensión de algo inmediato y previo como fundamento esencial del juicio lógico o de la operación matemática. Este interés por la intuición esencial lo repite Zubiri en otro trabajo suyo de juventud, Filosofía del ejemplo ${ }^{25}$ :

«A la inteligencia, como a los sentidos, le están inmediatamente dados los objetos. Y esta presencia inmediata de los objetos a la conciencia es lo que llamamos intuición. La función esencial del intelecto no es juzgar, sino tener objetos; encerrarlos o no en una fórmula predicativa, en una ley, será siempre un problema secundario ${ }^{26}$ \%.

Pareciera que toda la lógica del segundo decenio del siglo veinte resulta a Zubiri un titánico esfuerzo por fundamentar la inteligencia sometiéndola a leyes, a normas, leyes lógico-matemáticas ajenas a esa intuición esencial de los fenomenólogos o del intuicionismo de Brouwer. Fenomenología e intuicionismo exigían que la matemática se distanciara de la normatividad lógico simbólica. Aun reconociendo que con la logística se gana en rigor formal sin embargo, se corre el riesgo, según Zubiri, de recae en un nominalismo. Se puede, por tanto, prescindir de la utilidad técnica o pedagógica de la logística; el matemático no trabaja con símbolos sino con intuiciones.

Tanto el intuicionismo matemático como la filosofía fenomenológica son partidarios de la intuición frente al simbolismo. Pero esto, siendo mucho, no es todo. El intuicionismo de Brouwer había puesto en entre dicho el esfuerzo de Cantor por construir la matemática sobre las nociones de clase e infinito; en este aspecto, la lógica formal tradicional incumplía además uno de sus axiomas fundamentales. Sin extendernos en este complejísimo problema, cabe dejar indica-

${ }^{24}$ Archivo, oc. 11-2.

25 PE, 359-369.

${ }^{26} \mathrm{PE}, 336$. 
do simplemente que para Brouwer y Weil, la matemática tiene como característica determinar sus objetos indicando un número finito de operaciones que, efectivamente se puedan realizar. Finitismo e intuicionismo resultaban los dos capitales problemas metodológicos de la matemática contemporánea.

Pues bien, Brouwer interesó a Zubiri porque el intuicionismo había puesto en duda la validez del principio lógico denominado de tercio excluso que junto al de no-contradicción conforman no solamente la lógica aristotélica, sino además las direcciones simbólica y formalista contemporáneas. Y esto es decisivo. Deja escrito Zuburi:

«Lo característico de esta concepción [se refiere a la matemática cantoriana] es que se trata de una definición puramente lógica: se prescinde de que conozcamos o no la propiedad de que se trata. Basta con que la propiedad sea de tal índole que sostenga al principio de tercio excluso para que nos conste la definición y por tanto, la existencia del conjunto. Saber construirlo es otro problema muy interesante pero secundario. La matemática es así un sistema de ideas [...] Inspirándose sin duda en Poincaré, se levantan airados contra esta concepción Brouwer y Weyl. Los conjuntos infinitos, en efecto, no pueden ser dados más que por una ley que determine su propiedad característica ya que es imposible intentar una inducción completa que permitiese discernir si cada objeto pertenece o no al conjunto [...] el principio de tercio excluso se torna así en método de demostración ${ }^{27}{ }$.

La cuestión de fondo que se discute es que este viejo principio lógico aristotélico, no puede aplicarse a la matemática cantoriana del infinito; sucede además que, al igual que en el caso de Frege y de Russell, en la fundamentación de la matemática mediante la teoría de conjuntos se había acabado introducción nuevas y terribles paradojas. El resultado de todo esto, lo podemos seguir quizás con más claridad en algunos textos de Ortega y Gasset, por ejemplo en Las ciencias en rebeldía. Deja escrito Ortega:

«Pero donde más agudamente, aparece este nuevo temperamento científico es en la matemática. Su supeditación a la lógica había llegado en sus

27 Archivo, oc. 29. 
últimas generaciones hasta hacerse casi identidad. Pero he aquí que el holandés Brouwer descubre que el axioma lógico llamado del tercio excluso no vale para las entidades matemáticas y que es preciso hacer una matemática "sin lógica”, fiel a sí misma, indócil a axiomas forasteros» ${ }^{28}$.

Este mismo texto orteguiano se puede encontrar repetido, casi palabra por palabra, en varios textos posteriores casi siempre que Ortega cita a Brouwer; se trata, dice su autor, de renunciar valerosamente a la comodidad de presumir que la matemática es lógica y reconocer que lo único lógico es el pensamiento. Por tanto, la matemática presenta rasgos de ilogicismo como el asunto que Zubiri explica cuando explica la matemática cantoriana. Ortega y Zubiri; Zubiri y Ortega vinieron a pensar que el logicismo (así como la lógica formal tradicional) no son ya capaces de superar las fundamentadas críticas del intuicionismo de Brouwer y, en consecuencia, hallaron que la nueva lógica simbólica se mostraba resueltamente incompatible para la marcha de la filosofía contemporánea en general y para sus propios proyectos filosóficos muy en particular. Algo que, una vez tuvieran noticia de los resultados de Gödel, confirmarían de manera definitiva.

Es muy sencillo de comprobar que, frente al logicismo russelliano y frente al formalismo hilbertiano — del que a continuación nos ocuparemos- sólo el intuicionismo de Brouwer continuó apareciendo y citándose en las obras posteriores de Zubiri. Russell aparece y desaparece definitivamente en la tesis doctoral en el año veintitrés (1923) Hilbert aparece en Naturaleza, Historia y $\operatorname{Dios}^{29}$ y en otro curso inédito titulado Ciencia y Realidad pero solamente Brouwer continuó siendo tenido en cuenta por Zubiri hasta la trilogía final de su obra; de modo que nos reencontramos con el matemático holandés en Inteligencia y Logos:

«Los juicios de la matemática son pues juicios de algo real, juicios de lo real postulado. No son juicios acerca del ser posible sino juicios acerca de la realidad postulada. Esta conceptuación de la realidad matemática por construcción no es pues un axiomatismo formalista, pero tampoco es ni remotamente lo que se ha pensado como oposición rigurosa a este axiomatismo: el intuicionismo de, sobre todo Brouwer [...] Para el intuicionismo, cons-

\footnotetext{
${ }^{28}$ Ortega y Gasset, J.: Obras Completas. Tomo IV_1926/1931. Editorial Taurus. Fundación Ortega y Gasset. Madrid, 2005, pp. 334 y ss.

${ }^{29} \mathrm{NHD}, 306$.
} 
truir matemáticamente no es lo mismo que definir y construir conceptos. El intuicionismo rechaza la idea de que la matemática se funda en lógica; una demostración que apela al principio lógico del tercio excluso no es para Brouwer una demostración matemática» ${ }^{30}$.

Lo que sucede ahora, varías décadas más tarde, es que la matemática intuicionista no es ya sostenible por muchas razones, entre ellas, dice Zubiri, porque no podemos intuir conjuntos de cosas, ni siquiera si el conjunto fuera finito como quería Brouwer frente a Cantor. Un conjunto matemático es, para el maduro Zubiri, una realidad postulada por la razón. Muy lejos de aquella memoria de oposiciones a cátedra en Salamanca, afirma Zubiri que, en estricto rigor, no puede llamarse intuicionista a la matemática de Brouwer.

\section{El problema del formalismo: Hilbert y Zubiri}

Una vez catedrático en Madrid, nos encontramos con los primeros cursos universitarios de Zubiri. Eran los primeros años de la década de los treinta del siglo pasado. Entre ellos, contamos en el Archivo con el curso Fundamentos de Lógica ${ }^{31}$.

Sobre este curso en particular que, al parecer quedó incompleto, se ha especulado mucho; según se cuenta (y todo el mundo repite) Zubiri inició un seminario de lógica matemática sobre el manual de Hilbert-Akermann con muy reducido grupo de alumnos, aprovechando sus recientes estudios matemáticos con Zermelo. Así por ejemplo lo deja anotado Pintor Ramos ${ }^{32}$, quien conoce este dato a través del testimonio de D. Leandro Guevara. Y así lo recoge Vicente Muñoz Delga$\mathrm{do}^{33}$ quien además añade que Zubiri completó estando ya al corriente de los resultados de Gödel. Sin embargo, según se cuenta (y todo el mundo repite) Zubiri decidió interrumpirlo antes de su conclusión, convencido de su inutilidad para

${ }^{30}$ ZUBIRI, X.: Inteligencia sentiente. Inteligencia y Logos. Alianza Editorial. Fundación Xavier Zubiri. Madrid, 1982; pp. 139 y ss.

31 Archivo Signatura 0035006 Fundamentos de Lógica I. Curso de 19 lecciones de octubre a noviembre de 1935.

${ }^{32}$ PinTor Ramos, A.: Realidad y Verdad. Las bases de la filosofia de Zubiri. Publicaciones Universidad Pontificia de Salamanca. Salamanca, 1994; p. 184.

33 Muñoz Delgado, V.: Notas para la historia de la lógica durante la II República Española. Instituto Francisco Suárez del CSIC. Madrid, 1983; pp. 15 y ss. 
la empresa filosófica que el filósofo estaba buscando. Hay sin embargo quien encuentra que el curso es de una fecha diferente y en un ámbito académico distinto, me refiero a Velarde Lombraña ${ }^{34}$ que, en su descripción de la panorama de la lógica en España, muy desacertadamente afirma que el curso fue tras la guerra civil y que la victoria franquista consolidó una tradición neo-escolástica permitiendo tan solo que Gaos se dedicara a la fenomenología de Husserl. Del mismo curso programado y no impartido hace una referencia Vega Reñon ${ }^{35}$ quien lo supone como alternativa a los cursos de lógica de Julián Besteiro. Sin embargo, la fuente principal de todo este verdadero enredo historiográfico fue Manuel Mindán ${ }^{36}$ :

«La Introducción a la filosofía era dada por su titular don José Gaos. Pero también los profesores Zubiri y García Morente explicaban cursos de filosofía general, destinados principalmente a los que querían preparar su examen intermedio. Gaos daba además enseñanzas en relación con la Lógica. Comentó las Investigaciones de Husserl y [...] don Javier Zubiri tuvo asignado un curso sobre La nueva lógica formal... en el último curso $(1935-1936)^{37}{ }$.

Los cursos universitarios de Zubiri ${ }^{38}$ han estado inéditos hasta la publicación en dos mil siete (2007) del primero de los volúmenes — hay previsto un segundo en breve- dedicado a la labor docente e investigadora de Zubiri durante los primeros años de la década de los treinta, en la Facultad de Filosofía y Letras de la Universidad Central de Madrid. Zubiri, como es sabido, era catedrático de Historia de la Filosofía. Manuel García Morente era Decano y ambos impartían una Introducción a la Filosofía para el primer curso que ellos llamaban preparatorio.

En el curso 1931-1932 Zubiri impartió su curso de Introducción, un seminario sobre el Parménides de Platón (con la asistencia de María Zambrano) así como un curso sobre Introducción a la Fenomenología de Husserl recogido en Apuntes por Luis Felipe Vivanco. En el periodo 32-33 impartió Zubiri un largo curso con el título De Descartes a Hegel y unas once lecciones sobre Escoto y

34 Velarde Lombraña, J.: «Panorama de la lógica en España». Theoria - Segunda épocaVol. VII 1992, n. ${ }^{\circ}$ 16-17-18; Tomo A, pp. 339-345.

35 Vega ReÑón, L.: «La suerte de la lógica en la "Escuela de Madrid": notas sobre una desgracia». Revista de Filosofía. Vol. 28, Núm. 1 (2003): 33-58.

${ }^{36}$ Mindán Manero, M.: «El último curso de Ortega en la Universidad de Madrid». Revista de Filosofia (M) 16, 1957.

37 Oc., pp. $142-145$. 
Ockham; en el periodo de 1933-1934 Zubiri preparaba unas fichas sobre Fundamentos de Metafisica. Un seminario sobre la Lógica de Hegel. Finalmente, durante el curso académico 35-36, Zubiri sólo permanece en la universidad Central hasta diciembre del año1935. En este primer trimestre impartió un curso sobre Fundamentos de Lógica, del que se conservan dieciocho lecciones manuscritas de Zubiri y diez lecciones tomadas en el aula por María Araujo. En enero del treinta y seis Zubiri pide una excedencia y se marcha a Roma. Zubiri ya no volverá hasta el mes de noviembre del treinta y nueve. Finalmente y tras la victoria franquista, fue trasladado a Barcelona donde decidió abandonar su labor docente. ¿Fue ese curso sobre Fundamentos de Lógica al que alude Mindán Manero, Leandro Guevara y Muñoz Delgado (y todos los demás)? Mi respuesta es que no. Mi respuesta es que si alguna vez estuvo programado tal curso sobre el formalismo y el manual de Hilbert nunca se explicó. En contra de todos los testimonios anteriores, el curso de lógica al que aluden no existe. Nunca se impartió. Entre los materiales inéditos del Archivo no queda ninguna constancia de ello. Y por lo que respecta al texto de Hilbert, efectivamente se encuentra en la biblioteca personal de nuestro autor aunque no parece que Zubiri dedicara al libro alguna de sus densas lecturas, notas en los márgenes y subrayados. Decididamente el formalismo lógico de Hilbert no le interesó. Si, finalmente, se repasa el Programa Académico de la Facultad de Filosofía 1935-1936 39 de la Universidad Central de

38 ZubIRI, X.: Cursos universitarios VOL I. Alianza Editorial Fundación Xavier Zubiri. Madrid.2007.

39 Archivo Signatura 0036001 Programa de la Facultad de Filosofía y Letras Universidad Central de Madrid 1935-1936. En el índice de Enseñanzas Filosóficas de la Facultad de Filosofía aparece el APARTADO 1 Enseñanzas Filosóficas y dentro de este apartado, en el punto 5 titulado Lógica (página 44) dice:

5. LÓGICA. Doctor Besteiro, Catedrático (martes, jueves y sábados, a las once de la mañana).

a) Introducción a la Metodología de las ciencias prácticas (martes y jueves).

b) Los Principios filosóficos del Derecho y de la Política (continuación) (sábados).

Previamente en el punto 2 aparece lo siguiente:

2. FILOSOFÍA (Curso general). Doctor Zubiri Apalategui, Catedrático (lunes, miércoles y viernes de doce a una de la mañana) Lectura y Comentario de Suárez sobre el concepto de ente (viernes, de doce a una de la mañana).

Posteriormente dice:

12. HISTORIA DE LA FILOSOFÍA. Doctor Zubiri Apalategui. Catedrático (martes, jueves y sábados, de doce a una de la mañana) Parte descriptiva (genética y sistemática).

No aparece, por lo tanto, ningún curso sobre lógica formal según el manual de Hilbert-Akermann. 
Madrid puede comprobarse que efectivamente el encargado de la asignatura de Lógica era Besteiro y que no consta en ningún apartado, curso alguno ni seminario acerca de lógica formal, en ninguna de sus diferentes escuelas o corrientes contemporáneas del que estuviera encargado como docente Xavier Zubiri. Nada. Y abundando todavía más en este asunto, resulta que el contenido de estos Fundamentos de Lógica no puede estar más alejado de los temas, autores y problemas de la lógica formal de Hilbert; se trata del material de trabajo que Zubiri estaba elaborando para la publicación en la revista Cruz y Raya de un artículo titulado Filosofía y Metafisica ${ }^{40}$ y que comparte con dos artículos más: El saber filosófico y su historia y sobre todo ¿Qué es saber? Ambos forman parte de Naturaleza, Historia, Dios que se publicó, en su primera edición, en el año cuarenta y cuatro (1944).

La concepción de la Lógica que ofrece ahora Zubiri parece obedecer más a unas coordenadas si no opuestas, al menos, radicalmente diferentes de todos los otros trabajos anteriores. Su vinculación con respecto a los problemas de la fundamentación de la matemática es tajante; pareciera que Zubiri, siguiendo el impulso de la filosofía fenomenológica (Lógica Formal y Lógica Transcendental ${ }^{41}$ ) se hubiera propuesto volver a la raigambre fundamental del lógos, volver hacia la descripción del sentido propio de la lógica. Un vuelta hacia la primera indicación del concepto específico de la lógica, como también se encuentra en Heidegger (Lógica. La pregunta de la verdad ${ }^{42}$ ). Se trataría de regresar al comienzo, al principio originario a partir del cual surgieron las significaciones variadas de la palabra logos aunque buscando recuperar su formulación pre-lógica, su sentido previo como juicio o proposición lógico formal. Escribe Zubiri:

«La interna articulación de los elementos del logos es el objeto de la lógica. El problema del método se convierte así en "lógica", en una elaboración de la idea misma del logos; y, teniendo en cuenta que, la idea es la forma de las cosas, aquello que formalmente las constituye, se comprenderá que la

40 ZUBIRI, X.: Sobre el problema de la filosofía y otros escritos (1932-1944). Alianza Editorial Fundación Xavier Zubiri. Madrid, 2002; pp. 17-125.

${ }^{41}$ Husserl, E.: Lógica Formal y Lógica Transcendental. Ensayo de una crítica de la razón Lógica. Traducción de Villoro, Luis. U. N. A. M. Centro de Estudios Filosóficos. México, 1962. Véase también HuSSERL, E.: Experiencia y Juicio. Investigaciones acerca de la Genealogía de la Lógica. Traducción de J. ReuTER. U.N.A.M. México, 1980.

${ }^{42}$ Heidegger, M.: Lógica. La pregunta por la verdad. Versión española de J. A. CiRIA. Alianza Editorial. Madrid, 2004. 
lógica estudia lo que formalmente constituye el logos; y en este sentido eminentemente real es la lógica algo formal. De esta suerte, la lógica fue el organon del saber real, aquello que nos permite conquistar nuevas ideas y, con ello nuevos rasgos de las $\operatorname{cosas}^{43}{ }^{4}$.

\section{Conclusiones}

Presentamos por vez primera en este artículo la relación entre Zubiri y la recepción de las nuevas corrientes o direcciones que conforman la nueva lógica contemporánea. Hemos mostrado a través de algunos textos seleccionados de la primera etapa del pensamiento zubiriano que ya en la década de los años veinte del siglo pasado es notorio que el joven filósofo estuvo al tanto de la logística, representada por Russell, del intuicionismo de Brouwer y del formalismo de Hilbert. Con respecto al primero, Zubiri señala que nunca negó su valor y la ventaja que representa al dejar matemáticamente sistematizado una parte del razonamiento lógico; no obstante, considera que su valor es de utilidad y no de verdad y hay por qué aceptar que esta nueva concepción de la lógica tenga que ser toda la Lógica. Con respecto al intuicionismo, Zubiri reconoció siempre una dirección de la lógica contemporánea asimilable o, digamos, compatible con su proyecto filosófico de base netamente fenomenológica. Finalmente, en contra de todas las referencias anteriores, debe reconocerse que no hay constancia de que Zubiri impartiera un curso en la universidad acerca del formalismo lógico de Hilbert.

\section{Bibliografía}

Ayer, A. J. (1965). El positivismo lógico. México: Fondo de Cultura Económica.

Gómez IzQuierdo, A. (1917). Nuevas direcciones de la lógica. Madrid: Librería General de Victoriano Suárez.

- (1928). Análisis del pensamiento lógico. Granada: Facultad de Filosofía y Letras Universidad de Granada.

Heidegger, M. (2004). Lógica. La pregunta por la verdad. Versión española de J. A. CIRIA. Madrid: Alianza Editorial.

${ }^{43}$ NHD, 68. 
Husserl, E. (1962).Lógica Formal y Lógica Transcendental. Ensayo de una critica de la razón Lógica. Traducción de VILloro, Luis. México: Centro de Estudios Filosóficos. UNAM.

- (1980). Experiencia y Juicio. Investigaciones acerca de la Genealogía de la Lógica. Traducción de J. ReuTER. México: Centro de Estudios Filosóficos; UNAM

Kant, I. (1978). Crítica de la razón pura (Prólogo, Traducción, Notas e Índices de Pedro Rivas). Madrid: Ediciones Alfaguara.

Muñoz Delgado, V. (1983). Notas para la historia de la lógica durante la II República. Española. Madrid: Instituto Francisco Suárez del CSIC.

Ortega y Gasset, J. (2005). Obras Completas. Tomo IV, 1926/1931. Madrid: Editorial Taurus; Fundación Ortega y Gasset.

PinTOr Ramos, Antonio. (1994). Realidad y Verdad. Las bases de la filosofia de Zubiri. Salamanca: Publicaciones Universidad Pontificia de Salamanca.

Russell, B. (1983). Los principios de la Matemática. Madrid: Espasa-Calpe.

REY PASTOR, J. (1916). Introducción a la matemática superior. Madrid: Editorial Corona.

Mindán Manero, M. (1957). «El último curso de Ortega en la Universidad de Madrid». En Revista de Filosofía (M) 16.

Muñoz Delgado, V. (1962). Lógica matemática y lógica filosófica. Madrid (Carece de Editor).

- (1983). Notas para la historia de la lógica durante la II República Española. Madrid: Instituto Francisco Suárez del CSIC.

VEGA REÑón, L. (1996). Una guía de historia de la Lógica. Madrid: UNED.

- (2001). «La lógica en España (1890-1930): desencuentros». En Teorema. Revista internacional de Filosofía. VOL XX/1-2, pp. 21-38.

- (2003). "La suerte de la lógica en la "Escuela de Madrid": notas sobre una desgracia». En Revista de Filosofía. Vol. 28, pp. 33-58.

Velarde Lombraña, J. (1992). «Panorama de la lógica en España». En Theoria-Segunda Época- Volumen VII, n. ${ }^{\circ}$ 16-17-18; tomo A, pp. 339-345.

ZubIRI, X. (1982). Inteligencia Sentiente. Inteligencia y Logos. Madrid: Alianza Editorial; Fundación Xavier Zubiri.

- (1983): Inteligencia Sentiente. Inteligencia y Razón. Madrid: Alianza Editorial; Fundación Xavier Zubiri. 
- (1987). Naturaleza, Historia, Dios. Madrid: Alianza Editorial; Fundación Xavier Zubiri.

- (1999). Primeros Escritos (1921-1926). Madrid: Alianza Editorial; Fundación Xavier Zubiri.

- (2002). Sobre el problema de la filosofía y otros escritos (1932-1944). Madrid: Alianza Editorial; Fundación Xavier Zubiri.

- (2007): Cursos universitarios, VOL I .Madrid: Alianza Editorial; Fundación Xavier Zubiri.

Recibido: 18/11/2008

Aceptado: 10/01/2009 